\title{
ON THE INVARIANCE OF MALE OPTIMAL STABLE MATCHING
}

\author{
R. T. KUO and S. S. TSENG
}

Institute of Computer Science and Information Engineering National Chiao Tung University Hsinchu, Taiwan 30050, Republic of China.

\author{
Institute of Computer and \\ Information Science \\ National Chiao Tung University \\ Hsinchu, Taiwan 30050, Republic of China.
}

\begin{abstract}
.
The stable matching problem is that of matching two sets of agents in such a manner that no two unmatched agents prefer each other to their actual partners under the matching. In this paper, we present a set of sufficient conditions on the preference lists of any given stable matching instance, under which the optimality of the original male optimal stable matching is still preserved.
\end{abstract}

CR Categories: F.2.0, G.2.1.

Keywords: Stable matching problem, assignment, preference, invariance.

\section{Introduction.}

Gale and Shapley [1] introduced and solved the stable matching problem which is a kind of assignment game problem. That problem involves two disjoint sets of equal cardinality $n$, the men and the women. Each person ranks all members of the opposite sex in order of preference. A stable matching is defined as a complete matching between men and women with the property that no man and woman who are not partners both prefer each other to their actual partners under the matching.

Several stable matching algorithms $[1,5,6,7]$ were proposed to solve the problem by returning the male (or female) optimal stable matching as its answer. Any sequential one of those algorithms will be called a Stable-Matching Algorithm in this paper.

The original stable matching problem has a restriction on the static requirements of the preference lists. The purpose of this paper is to relax this requirement by allowing the dynamic adjustment of the stable matching problem instance after the male optimal stable matching has been found. This would lead to the dynamic stable matching problem. Note that the male optimal stable matching for the adjusted stable matching instance is the solution of the problem. The purpose of this paper is

Received August 1989. Revised April 1990. 
not to find a solution for this problem. Our main concern is: What kind of alterations can be made to the preferences so that the male optimal stable matching for the resulting instance is identical to the male optimal stable matching for the original? That is, what conditions will lead to the invariance of the male optimal stable matching?

In Section 2 we recall briefly the definitions and some background results of the stable matching problem; in Section 3 we show a set of sufficient conditions on the preference lists, under which the male optimal stable matching is preserved. In Section 4 concluding remarks will be given.

\section{Definitions and background results.}

An instance of the stable matching problem consists of a set $M$ of $n$ men and a set $W$ of $n$ women. Each member in these two sets has a total rank-ordered preference list of the $n$ people of the opposite sex. For convenience, let $P\left(m_{i}\right)$ and $P\left(w_{j}\right)$ denote the preference lists for any man $m_{i}$ and any woman $w_{j}$, respectively. When no confusion will occur, $P_{i}$ will sometimes be used to represent $P\left(m_{i}\right)$ or $P\left(w_{i}\right)$. Let $P(M)$ and $P(W)$ denote the sets of preference lists for all members in $M$ and in $W$, respectively. Let the ranking function $r\left(m_{i}, w_{j}\right)$ denote the position of woman $w_{j}$ in $P\left(m_{i}\right)$ and $r\left(w_{j}, m_{i}\right)$ denote the position of man $m_{i}$ in $P\left(w_{j}\right)$. For each man $m_{i}$, let $w_{j} \succ_{i} w_{k}$ denote that $m_{i}$ prefers woman $w_{j}$ to woman $w_{k}$. Similarly, for each woman $w_{i}$, let $m_{j} \succ_{i} m_{k}$ denote that $w_{i}$ prefers $\operatorname{man} m_{j}$ to $\operatorname{man} m_{k}$. Note that the statement " $m_{i}$ prefers $w_{j}$ to $w_{k}$ to $w_{l}$ " can be written as $w_{j} \succ_{i} w_{k} \succ_{i} w_{l}$ or $m_{i}: w_{j}>w_{k}>w_{l}$ or more simply $m_{i}: w_{j} w_{k} w_{l}$. Let the preference pattern $P=(P(M), P(W))=\left(P\left(m_{1}\right), \ldots, P\left(m_{n}\right)\right.$, $\left.P\left(w_{1}\right), \ldots, P\left(w_{n}\right)\right)$ denote the vector of preference lists of all members in $M \cup W$, and let $(M, W ; P)$ denote a stable matching problem instance.

A matching $\mu$ is a one-to-one mapping of the men and the women, i.e., an invertible function $\mu: M \rightarrow W$ such that $\mu\left(m_{i}\right)$ is the woman matched with man $m_{i}$ and $\mu^{-1}\left(w_{j}\right)$ is the man matched with woman $w_{j}$. The pair $(m, w)$ blocks the matching $\mu$ if $m$ and $w$ are not matched by $\mu$ but prefer each other to their respective partners given in $\mu$. A matching is stable if it is not blocked by any pair. A matching that is not stable is called unstable.

The algorithm proposed by Gale and Shapley [1] finds the Male Optimal Stable Matching (MOSM) $\mu_{m}$, although it can, by relabeling, also find the female optimal stable matching. Note that the sequential Stable-Matching algorithms for a solution to a stable matching instance $[1,5,6]$ are based on a sequence of proposals from the men to the women. It is shown [5] that the sequence of proposals ends with every woman holding a unique proposal, and that the proposals held constitute a stable matching which is the MOSM. A similar outcome results if the roles of males and females are reversed, in which case the resulting stable matching that is the female optimal stable matching may or may not be the same as that obtained from the male proposal sequence. It is pointed out in [5] that the MOSM is female pessimistic, i.e., 
for every woman $w$, there is no stable matching in which $w$ is matched to a man she prefers less than her partner in the MOSM. Similarly, the female optimal stable matching is male pessimistic. In other words, the achievement of best potential partners by the members of one sex results in the members of the opposite sex having their worst potential partners.

Two fundamental implications of the male proposal sequence, implicit in [5], are

(i) if $m$ proposes to $w$ then there is no stable matching in which $m$ has a better partner than $w$, and

(ii) if $w$ receives a proposal from $m$ then there is no stable matching in which $w$ has a worse partner than $m$.

From these observations, it is shown [3] that we should explicitly remove $m$ from $w$ 's list, and $w$ from $m$ 's, if $w$ receives a proposal from someone she likes better than $m$. Note that when man $m$ is temporarily accepted by woman $w$, all the men in woman $w$ 's preference list who are lower than man $m$ can be eliminated. The resulting lists are referred to as the male-oriented shortlists, for the given problem instance.

In the context of the male-oriented shortlists, a male-oriented rotation $[3,4]$ exposed in $\mu$ is a sequence

$$
r=\left(m_{0}, w_{0}\right), \ldots,\left(m_{p-1}, w_{p-1}\right)
$$

of pairs from $\mu$ such that, for each $i(0 \leq i \leq p-1)\left(\right.$ i) $w_{i}$ is first in $m_{i}$ 's shortlist, and (ii) $w_{i+1}$ is second in $m_{i}$ 's shortlist ( $i+1$ taken modulo $p$ ).

Similarly, we can, by relabeling, also define the female-oriented rotation exposed in $\mu^{-1}$. Note that for a given matching there may be many or there may be no exposed rotations.

It is pointed out in [3] that if the first entries in the male-oriented shortlists do not specify the $\mu_{w}$, then at least one rotation must be exposed. The main significance of such a rotation lies in the fact that if, in $\mu_{m}$, each $m_{i}$ exchanges his partner $w_{i}$ for $w_{(i+1) \bmod p}$ then the resulting matching is also stable. If, given the rotation $r=\left(m_{0}, w_{0}\right), \ldots,\left(m_{p-1}, w_{p-1}\right)$, each successor $y$ of $m_{i-1}$ in $w$ 's shortlists is removed, together with the corresponding appearance of $w_{i}$ in $y$ 's list, for each $i(0 \leq i \leq p-1, i-1$ taken modulo $p)$, then the rotation $r$ is said to have been eliminated. This process is referred to as eliminating a rotation. It is proved in [3] that every stable matching for a given stable matching instance may be obtained from the shortlists by eliminating some sequence of zero or more exposed rotations.

Let us briefly review the Gale-Shapley algorithm $[1,2]$ here.

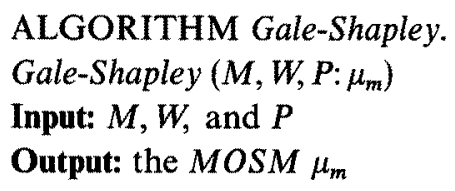

At the start of the algorithm, each person is free and becomes engaged during the execution of the algorithm. Once a woman is engaged she never becomes free again, 
but men can alternate between being free and being engaged. Repeating the following until all men are engaged:

Choose a free man $m$, and have $m$ propose to his most preferred woman $w$ on his preferences list, such that $w$ has not already rejected $m$. If $w$ is free, then $w$ and $m$ become engaged. If $w$ is engaged to man $m^{\prime}$ (a conflict occurs), then she rejects the man ( $m$ or $m^{\prime}$ ) that she least prefers, and becomes, or remains, engaged to the other man. The rejected man becomes, or remains, free.

\section{The invariance of the male optimal stable matching.}

In view of the dynamization on a given preference pattern, we may ask whether the corresponding male optimal stable matching is still preserved for the new preference pattern. This gives the motivation for finding the conditions on the preference lists which will lead to the invariance of the male optimal stable matching.

In the following we will answer the question: Is the MOSM invariant when the male and female preference lists are subject to random permutations without crossover? In other words, can we permute the sublists on either side of the MOSM on the male or female preference lists?

It is clear that the sublists to the right of the MOSM in the male preference lists and to the left of the MOSM in the female preference lists cannot effect the MOSM since they play no part in the Gale-Shapley algorithm. They therefore can be permuted at will.

As will be shown in the following example, the alterations to the right of the MOSM in the female preference lists can cause the MOSM to change.

ExAmPLE. Given $(\{1, \ldots, 8\},\{1, \ldots, 8\} ; P)$ with the preference pattern $P$ defined by

M: Male's preference lists

1: $\begin{array}{llllllll}5 & 8 & 2 & 3 & 1 & 4 & 7 & 6\end{array}$

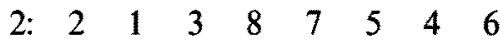

3: $\begin{array}{llllllll}7 & 3 & 1 & 4 & 8 & 6 & 2 & 5\end{array}$

4: $\quad 4 \quad 7 \quad \begin{array}{lllllll} & 4 & 3 & 6 & 2 & 5 & 8\end{array}$

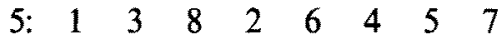

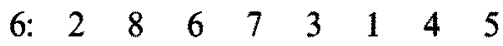

$\begin{array}{lllllllll}7: & 5 & 3 & 4 & 7 & 1 & 6 & 8 & 2\end{array}$

$\begin{array}{lllllllll}8: & 4 & 8 & 2 & 5 & 7 & 6 & 3 & 1\end{array}$
$W$ : Female's preference lists

$\begin{array}{lllllllll}\text { 1: } & 6 & 1 & 8 & 7 & 4 & 3 & 2 & 5 \\ \text { 2: } & 3 & 7 & 4 & 5 & 8 & 2 & 6 & 1 \\ \text { 3: } & 8 & 6 & 1 & 2 & 7 & 4 & 3 & 5 \\ \text { 4: } & 2 & 1 & 6 & 7 & 8 & 3 & 5 & 4 \\ \text { 5: } & 4 & 3 & 8 & 5 & 1 & 2 & 7 & 6 \\ \text { 6: } & 7 & 4 & 5 & 3 & 6 & 1 & 2 & 8 \\ \text { 7: } & 1 & 7 & 6 & 8 & 2 & 4 & 5 & 3 \\ \text { 8: } & 6 & 5 & 1 & 4 & 2 & 3 & 7 & 8 .\end{array}$

With the given instance, the $\operatorname{MOS} M \mu_{m}$ is

$$
\{(1,3),(2,7),(3,6),(4,1),(5,2),(6,8),(7,4),(8,5)\} \text {. }
$$


Suppose that the male preference pattern is left alone and the female preference pattern is changed to:

$\begin{array}{lllllllll}\text { 1: } & 6 & 1 & 8 & 7 & 4 & 2^{*} & 3^{*} & 5 \\ \text { 2: } & 3 & 7 & 4 & 5 & 8 & 6^{*} & 2^{*} & 1 \\ \text { 3: } & 8 & 6 & 1 & 2 & 7 & 4 & 5^{*} & 3^{*} \\ \text { 4: } & 2 & 1 & 6 & 7 & 8 & 3 & 5 & 4 \\ \text { 5: } & 4 & 3 & 8 & 5 & 7 * & 1^{*} & 2^{*} & 6 \\ \text { 6: } & 7 & 4 & 5 & 3 & 6 & 1 & 2 & 8 \\ \text { 7: } & 1 & 7 & 6 & 8 & 2 & 4 & 5 & 3 \\ \text { 8: } & 6 & 5 & 1 & 4 & 2 & 3 & 7 & 8 .\end{array}$

Note that only those figures marked with *'s are changed from the original. The $\operatorname{MOSM}$ for the resulting instance becomes $\{(1,8),(2,1),(3,6),(4,7),(5,3),(6,2),(7,5)$, $(8,4)\}$.

All that remains is to show that the permutations to the left of the MOSM in the male preference lists leave the MOSM invariant.

THEOREM. Given any arbitrary instance $(M, W ; P)$ and its $M O S M \mu_{m}$, the interchange operation applied on a sublist to the left of the MOSM in the male preference lists cannot effect the MOSM.

Proof. We first show that such interchange leave the MOSM as a stable matching. This is straightforward because any pair which caused instability in the new instance would have caused instability in the original MOSM and this is a contradiction because the original MOSM is stable.

Next, we claim that there is no female-oriented rotation exposed in the original MOSM in the female preference pattern of new instance. Suppose, to the contrary, that there exists such a rotation $r$. By the definition of the new instance, the rotation $r$ must also be a female-oriented rotation exposed in MOSM in the female preference pattern for $(M, W ; P)$. This implies that the better potential partners of the male could be achieved by the elimination of $r$ from the female-oriented shortlists corresponding to the $M O S M$ in the female preference pattern for the $(M, W ; P)$, and contradicts that $\mu_{m}$ is the $\operatorname{MOSM}$ for $(M, W ; P)$. Thus there does not exist any female-oriented rotation exposed in the original MOSM for the female preference pattern of the new instance. Combining this with our claim that the original MOSM is stable for the new instance, we conclude that the original MOSM is also the MOSM for the new instance.

The proof is now complete.

COROLlary. Given any arbitrary instance $(M, W ; P)$ and its $\operatorname{MOSM} \mu_{m}$, any permutation of a sublist to the left of the MOSM in the male preference lists cannot effect the MOSM. 
Proof. It is well known that a permutation can be made up of a sequence of appropriate interchanging operations. By repeatedly applying the theorem above we can easily show that the permutation of a sublisst to the left of the MOSM in the male preference lists leave the MOSM invariant. Thus, the corollary follows.

Further, by repeated application of the above corollary, it is easy to show that the permutations to the left of the MOSM in the male preference lists leave the MOSM invariant.

\section{Concluding remarks.}

In this paper, we have presented a set of sufficient conditions on the preference lists of any given stable matching instance, under which the optimality of the original male optimal stable matching is still preserved. The following is an outline of our results.

(1) The permutations of the sublists to the left of the MOSM in the male preference lists cannot effect the MOSM.

(2) The permutations of the sublists to the right of the MOSM in the male preference lists cannot effect the MOSM.

(3) The permutations of the sublists to the left of the MOSM in the female preference lists cannot effect the MOSM.

(4) The permutations of the sublists to the right of the MOSM in the female preference lists can cause the MOSM to change.

Note that the optimality-preserving conditions given in this paper are useful in the study of the stable matching problem with dynamic preference orderings.

In closing this remark, let us pose two interesting problems. The first one is an extension of the results given in this paper, i.e. the consideration of the effects of crossover on the invariance of the MOSM. The second one is stated as follows: Given any stable matching instance $l=(M, W ; P)$ and its $M O S M \mu_{m}$, does there exist any coalition $C$ of members from $M$ such that

$$
\begin{array}{ll}
\sum_{m \in C}\left[r\left(m, \mu_{m}(m)\right)-r\left(m, \mu_{m}^{\prime}(m)\right)\right] & >0, \\
r\left(m, \mu_{m}(m)\right) \geq r\left(m, \mu_{m}^{\prime}(m)\right), & \forall m \in C, \text { and } \\
r\left(m, \mu_{m}(m)\right)=r\left(m, \mu_{m}^{\prime}(m)\right), & \forall m \in M-C
\end{array}
$$

under the conditions that some members in $C$ give false preferences, where $\mu_{m}^{\prime}$ is the $M O S M$ for the instance corresponding to the falsified preference pattern? How identify one, if it exists? Note that the results given in this paper have shown that there is no effect on the MOSM if those members in $C$ give their falsified preferences satisfying the optimality-preserving conditions. 


\section{Acknowledgement.}

The authors are grateful for the valuable and constructive comments made by the anonymous referees.

\section{REFERENCES}

1. D. Gale and L. S. Shapley, College admissions and the stability of marriage, Amer. Math. Monthly 69 (1962), pp. 9-15.

2. D. Gusfield, Three fast algorithms for four problems in stable marriage, SIAM J. Comput. Vol. 16, No. 1, Feb. 1987, pp. 111-128.

3. R. W. Irving and P. Leather, The complexity of counting stable marriages, SIAM J. Comput., Vol. 15, 1986, pp. 655-667.

4. R. W. Irving, P. Leather and D. Gusfield, An efficient algorithm for the optimal stable marriage, J. ACM, Vol. 34, No. 3, July 1987, pp. 532-543.

5. D. G. McVitie and L. B. Wilson, The stable marriage problem, Communication of the ACM, Vol. 14, No. 7, July 1971, pp. 486-490.

6. D. G. McVitie and L. B. Wilson, Algorithm 411, Pts. 1-3. Three procedures for the stable marriage problem, Communication of the ACM, Vol. 14, No. 7, July 1971, pp. 491-492.

7. S. S. Tseng and R. C. T. Lee, A parallel algorithm to solve the stable marriage problem, BIT 24,1984 , pp. 308-316. 